\title{
Adsorption of Ibuprofen and Dopamine on Functionalized Gold Using Surface Plasmon Resonance Spectroscopy at Solid-Liquid Interface
}

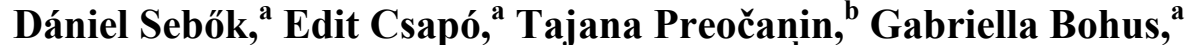 \\ Nikola Kallay, ${ }^{\mathrm{b}}$ and Imre Dékány ${ }^{\mathrm{a}, \mathrm{c}, *}$ \\ ${ }^{a}$ MTA-SZTE Supramolecular and Nanostructured Materials Research Group of the Hungarian Academy of Sciences, \\ H-6720 Dóm sqr. 8., Szeged, Hungary \\ ${ }^{\mathrm{b}}$ Department of Chemistry, Faculty of Science, University of Zagreb, HR-10000, Horvatovac 102a, Zagreb, Croatia \\ ${ }^{\mathrm{c}}$ Department of Medical Chemistry, Faculty of Medicine, University of Szeged, H-6720 Szeged, Dóm sqr. 8. Hungary
}

RECEIVED AUGUST 9, 2013; REVISED SEPTEMBER 22, 2013; ACCEPTED OCTOBER 10, 2013

\begin{abstract}
Ultrathin gold nanofilms $(\approx 50 \mathrm{~nm})$ are suitable for detection of adsorbed molecules at solidliquid interface of various sizes due to their surface plasmon resonance (SPR) properties. This SPR technique makes it possible to study the surface adsorption in nanomol range amount on gold surfaces of a maximum of one $\mathrm{mm}^{2}$ and to determine the adsorbed amount as a function of equilibrium concentration. Adsorption of L-cysteine, L-glutathione, ibuprofen and dopamine on the gold surface was examined. Moreover, the binding capability of ibuprofen and dopamine molecules on the gold surface functionalized by L-cysteine and L-glutathione was studied as well. Adsorption isotherms were recorded using the flow measuring technique, which allows determination of the amount of adsorbed material even in the $\mathrm{nmol} / \mathrm{cm}^{2}$ order of magnitude, the cross sectional areas of adsorbed molecules. The adsorption enthalpies (isosteric heat of adsorption) were determined from adsorption isotherms at different temperatures. The surface orientations of the studied molecules were analyzed by MarvinSketch program. (doi: $10.5562 / \mathrm{cca} 2343)$
\end{abstract}

Keywords: surface plasmon resonance, adsorption, functionalization, L-cysteine, L-glutathione, ibuprofen, dopamine, gold thin film

\section{INTRODUCTION}

Since the first report on the phenomenon of surface plasmon resonance (SPR) (studies on processes taking place on metal surfaces and the detection of gases), appliances have undergone an enormous development regarding both technique and applications. Since the first presentation of a surface plasmon resonance (SPR) gas sensor ${ }^{1,2}$ the scientific attention focusing on these instruments has been undiminished. SPR sensors have become important means for the qualitative and quantitative characterization of biomolecular interactions. Development of SPR devices serving for studies on chemical and biological analytes is pursued intensively, so that number of publications reporting studies on interactions of importance for medical diagnostics, environmental protection, bacteriology and food quality increases rapidly. In the last three decades SPR devices have become some of the rifest and most popular gas $^{3,4}$ and label-free biosensors ${ }^{5-7}$ due to their outstanding sensitivity and their easy use and evalua- tion. The earliest documentation of metal-light interaction anomalies comes from $\mathrm{Wood}^{8}$ and Fano; ${ }^{9}$ these observations were followed by those by $\mathrm{Otto}^{10}$ and Kretschmann ${ }^{11}$ in 1968 , who described that in several attenuated total reflection configurations the shape of the reflection curve is caused by the excitation of surface plasmons. Since that time the application methods and technical solutions have developed continually: there are instruments using prism, ${ }^{12}$ grating $^{13}$ and waveguide coupler, ${ }^{14}$ while on the other hand there are angle ${ }^{15}$ or wavelength ${ }^{16}$ modulating and imaging ${ }^{17,18}$ devices. The most popular configuration is the socalled Kretschmann-configuration (Figure 1), in which the momentum of incident light is coupled to the free oscillations of the conduction electrons at a metal surface through a prism in order to increase the wavenumber of light, thus generating the conditions of surface plasmon resonance.

The propagation constant of the surface plasmon $\left(k_{\mathrm{SP}}\right)$ at the metal-dielectric interface is

\footnotetext{
* Author to whom correspondence should be addressed. (E-mail: i.dekany@chem.u-szeged.hu)
} 


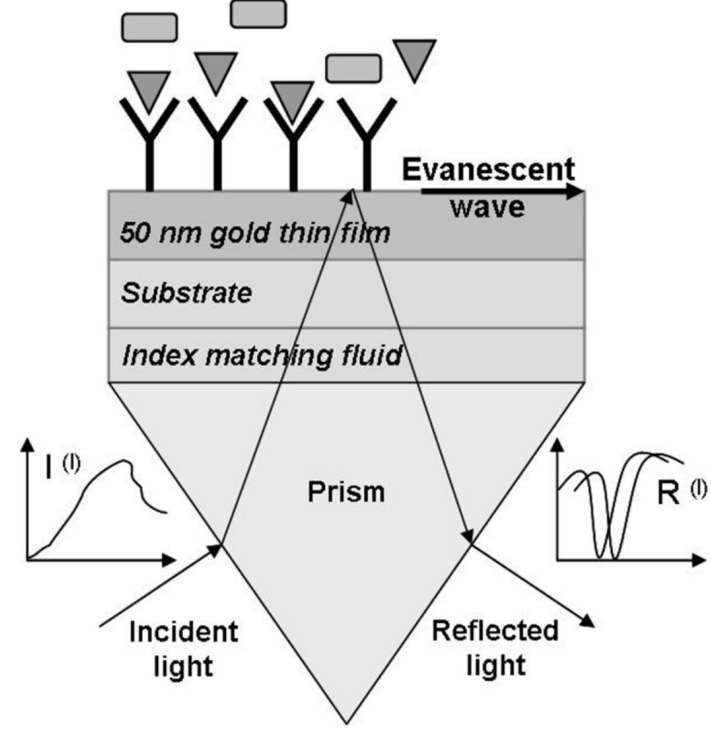

Figure 1. Schematic representation of prism coupling (Kretschmann-configuration) in SPR technique. ${ }^{14}$

$$
k_{\mathrm{SP}}=\frac{2 \pi}{\lambda} \sqrt{\frac{\varepsilon_{\mathrm{d}} \varepsilon_{\mathrm{m}}}{\varepsilon_{\mathrm{d}}+\varepsilon_{\mathrm{m}}}}
$$

where $\lambda$ is the wavelength in vacuum, $\varepsilon_{\mathrm{d}}$ and $\varepsilon_{\mathrm{m}}$ are the complex permittivities of dielectric and metal, respectively. ${ }^{17}$ The light wave cannot be coupled directly, because the real part of the surface plasmon's propagation constant is larger than the wavenumber of the light's wave vector that is parallel to the surface. The surface plasmon can be excited if the light's wavenumber is increased by a prism having a proper refractive index (Eq. 2).

$$
\frac{2 \pi}{\lambda} n_{\mathrm{p}} \sin (\vartheta)=\operatorname{Re}\left(k_{\mathrm{SP}}\right)
$$

where $n_{\mathrm{p}}$ is the refractive index of the prism (larger than the refractive index of the dielectric $n_{\mathrm{p}}$ ) and $\theta$ is the angle of incidence. Thus, scanning the angle with a polarized monochromatic incident light (angle modulation) or scanning the wavelength of polychromatic light at a fixed angle of incidence (wavelength modulation) the matching condition can be achieved, and the surface plasmon can be excited by an evanescent wave propagating along the metal-dielectric interface. It has to be noted that in case of waveguide or grating couplers the propagating constant of waveguide mode or the momentum of diffracted light has to fulfill the matching conditions. $^{18}$

Chemical or physical adsorption onto the gold surface changes the refractive index of the dielectric, thereby the propagation constant of the surface plasmon. Therefore, in order to fulfill the matching conditions in
Eq. (2), $\lambda$ or $\theta$ has to change. By measuring one of these values (while the other is fixed), the change in the refractive index of the dielectric can be calculated, and the concentration of analyte molecules on the surface can be determined.

Nowadays the importance of gold nanoparticles (AuNPs) is increasing in several biological and medical applications, because they have a neutral physiological effect, and their expediency in many types of applications such as CT and X-ray contrast materials, ${ }^{19,20}$ photothermal therapy, ${ }^{21,22}$ drug delivery ${ }^{23,24}$ and plasmonic properties ${ }^{25,26}$ has been proven. Most of the applications require surface functionalization, either in order to bind proteins, drugs (that do not contain thiol groups) onto the surface of AuNPs or to find the target molecule/group in the course of targeted drug or contrast material delivery.

Several amino acids are suitable to functionalize the surface of gold particles. In this work the adsorption of ibuprofen and dopamine drug molecules onto Lcysteine and L-glutathione modified gold surface has been investigated.

\section{Application of SPR Sensors in Biological Research}

Surface plasmon resonance (SPR) sensors are suitable for application in studies on biological interactions due to their sensitivity, their resolution and their material requirement. Since these devices essentially function as refractometers, i.e. they measure changes in refractive index (see Eq. 3), their sensitivity and resolution are most often specified in refractive index units (RIU). After suitable calibration, it can be converted to surface concentration expressed as amount ("number of moles") of adsorbed species per surface area (e.g. in nmol cm${ }^{-2}$ ) using the following expression ${ }^{17}$

$$
\Delta n=\left(\frac{\mathrm{d} n}{\mathrm{~d} c}\right) \frac{\Gamma}{h}
$$

where $\mathrm{d} n / \mathrm{d} c$ is the dependency of refractive index on concentration of adsorbent in the solution (typically ${ }^{25,26}$ $\left.0.1-0.3 \mathrm{mg} \mathrm{L}^{-1}\right), h$ is the adsorption layer thickness and $\Gamma$ denotes surface concentration, expressed as amount ("number of moles") of adsorbed species per surface area.

The conventional adsorption measuring technique at solid-liquid interface is not acceptable for characterization of our studied systems because the preparation of biomolecules functionalized surface is very expensive. Among the surface plasmon resonance technique the other possible two-dimension method for characterization of adsorption is the optical waveguide lightmode spectroscopy (OWLS). Similar to SPR, this OWLS technique also require very small amount of chemical agent and the sensitivity is very high as well. 
Before functionalization of nanoparticles the binding capacity i.e. the cross-sectional area of the studied adsorbed molecules has to be determined by interpretation of the respective adsorption isotherms.

\section{MATERIALS AND METHODS}

\section{Materials}

The following chemicals were used in the experiments: L-cysteine (L-Cys) (Fluka $\geq 99.5 \%$ ), L-glutathione (LGSH) (Fluka $\geq 97 \%$ ), ibuprofen (Sigma-Aldrich, $\geq 98$ $\%$ ), dopamine (Sigma-Aldrich,). All of the solutions were prepared using Milli-Q ultrapure water.

\section{Surface Plasmon Resonance (SPR) Measurements}

SPR measurements were carried out in order to determine the adsorbed amounts and the monomolecular coverage of L-cysteine (L-Cys), L-glutathione (L-GSH), ibuprofen and dopamine on the (pure) gold surface, as well as adsorption of ibuprofen and dopamine on the gold surface functionalized with L-cysteine and Lglutathione. A two-channel SPR sensor platform developed at the Institute of Photonics and Electronics (Prague) was applied. The SPR chip is a thin gold layer (50 $\mathrm{nm}$ thickness), deposited on a glass substrate. During measurements the flow rate of $30 \mu \mathrm{L} \mathrm{min}{ }^{-1}$ was used, and the temperature was kept at $20^{\circ} \mathrm{C}$. In all cases three parallel measurements were carried out. In order to test the reversibility of the adsorption process, the pure water was applied after the adsorption step. The recorded spectra were analyzed in real-time by a special software package that allows determination of the resonant wavelength in both sensing channel.

\section{RESULTS}

\section{Adsorption on Pure Gold Surface Thin Films}

Figures 2 and 3 represent the plasmonic curves of adsorption of L-Cys and L-GSH in a flow system on the surface of a gold SPR chip. Cysteine and glutathione were immobilized on the surface of SPR gold chips from their aqueous solutions in the concentration range 0.01-10 mmol dm${ }^{-3}$. Surface concentrations were plotted as a function of time. Two steps of the experiments were presented. In the first step lower concentrations were applied followed by washing with pure water, then in the second step higher concentrations were introduced, again followed with washing with pure water. As it could be seen from Figures 2 and 3, after washing with water the adsorption amount was not reduced to zero, so that one can conclude that the adsorption process of L-Cys and L-GSH is not fully reversible. This means that substantial part of the adsorbed amount re-

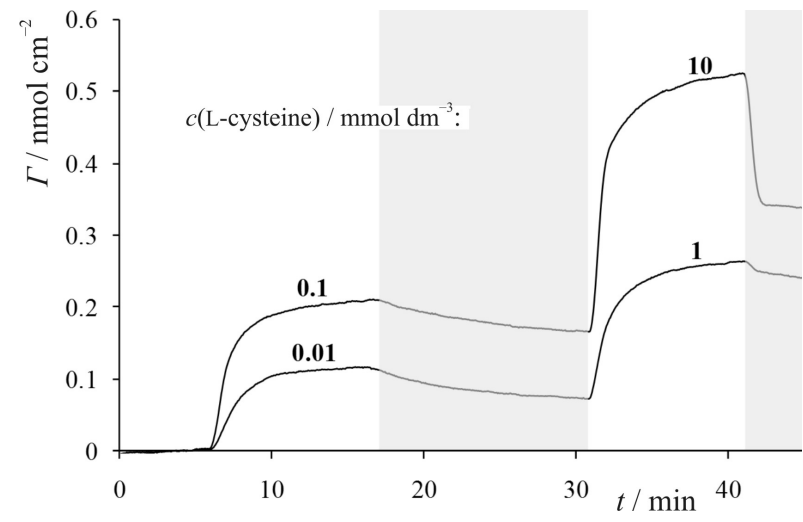

Figure 2. Representative SPR curves (sensorgrams) of adsorption of L-cysteine on the gold surface from aqueous solutions. Washing with water is denoted by gray areas.

mains irreversibly bound at the gold surface. It should be noted that this irreversibly bound amount increases with concentration of adsorbent, but at higher concentrations (above $1 \mathrm{mmol} \mathrm{dm}^{-3}$ ) approaches its maximum value.

Figure 4 represents adsorption isotherms for L-Cys and L-GSH on the gold surface. Data for both total and irreversibly bound adsorbates are presented.

It could be concluded that irreversibly bound LCys and L-GSH reaches certain maximum concentration, which holds especially for L-GSH. The area occupied by adsorbed molecule $a_{\mathrm{m}}$ could be calculated from monomolecular adsorbed amount, e.g. by

$$
a_{\mathrm{m}}=\frac{1}{N_{\mathrm{A}} \Gamma_{\mathrm{m}}}
$$

where $\Gamma_{\mathrm{m}}$ is monolayer surface concentration, and $N_{\mathrm{A}}$ is the Avogadro constant. In numerical form, introducing

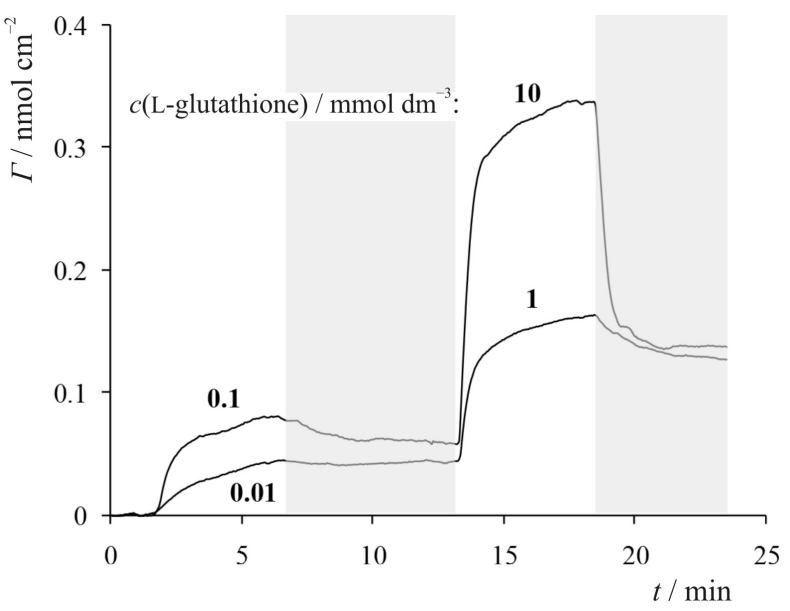

Figure 3. Representative SPR curves (sensorgrams) of adsorption of L-glutathione on the gold surface from aqueous solutions. Washing with water is denoted by gray areas. 


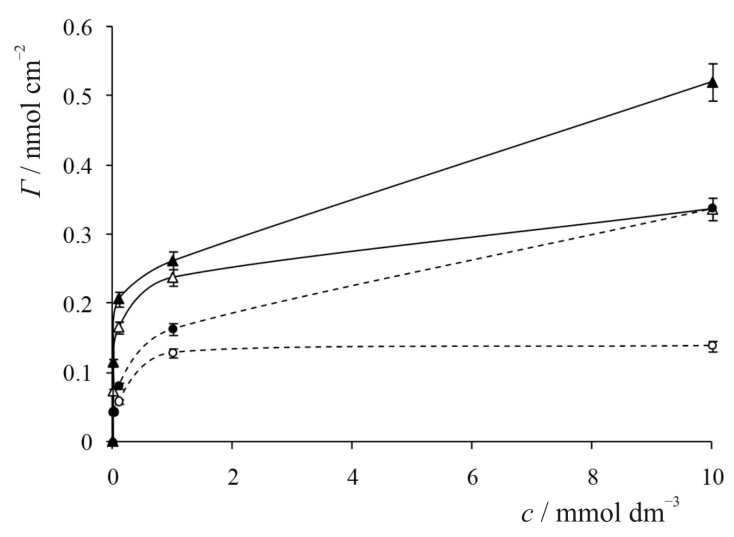

Figure 4. Adsorption isotherms for L-cysteine $(\boldsymbol{\Delta}, \Delta)$ and Lglutathione $(\bullet, \circ)$ on gold surface from aqueous solutions: total adsorption (full symbols) and irreversible adsorption (open symbols). The error of $\Gamma$ values is $\pm 5.2 \%$.

suitable units, Eq. (4) reads

$$
a_{\mathrm{m}} / \mathrm{nm}^{2}=0.166 \times \frac{1}{\Gamma_{\mathrm{m}} / \mathrm{nmol} \mathrm{cm}}
$$

The results are summarized in Tables 1 and 2.

Adsorption of ibuprofen and dopamine on the (pure) gold surface is presented in Figure 5. Both experiments consisted of 6 subsequent steps, each one corresponding to higher adsorbent concentration. The washing procedure was applied after final step, i.e. after adsorption

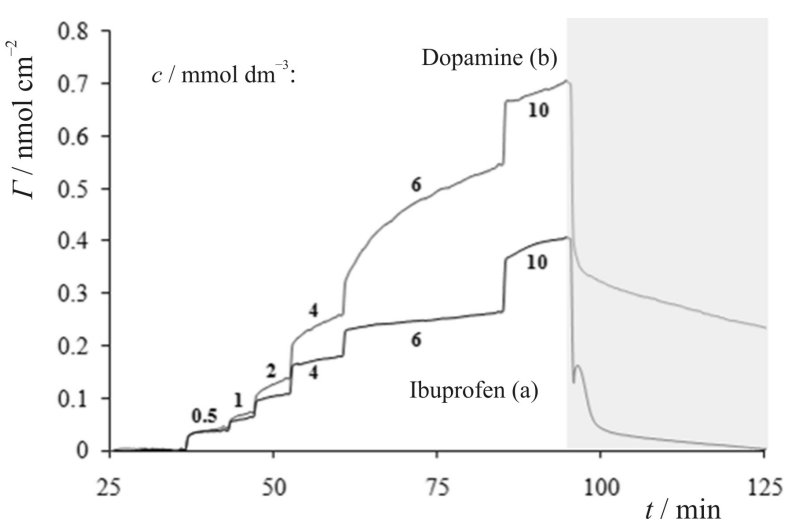

Figure 5. Representative SPR curves of adsorption of ibuprofen (a) and dopamine (b) on gold surface from aqueous solutions at different concentrations $(0.5,1,2,4,6,10 \mathrm{mmol}$ $\left.\mathrm{dm}^{-3}\right)$. Washing with water is denoted by gray area.

from highest adsorbent concentration of $10 \mathrm{mmol} \mathrm{dm}^{-3}$. Figure 6 presents respective adsorption isotherms.

The adsorbed amount increases with adsorptive concentration. Washing step reduced rapidly adsorbed amount of ibuprofen to zero so that one may conclude that adsorption of these species is fully reversible. Dopamine showed reduction of surface concentration in aqueous environment but this process is rather slow and will probably reach zero surface coverage after prolonged washing procedure.

Adsorption results were analyzed by applying the linear form of the Langmuir adsorption isotherm, i.e. by

Table 1. The monolayer adsorption capacities $\left(\Gamma_{\mathrm{m}}\right)$ and molecular cross sectional areas $\left(a_{\mathrm{m}}\right)$ on gold surface for different bioconjugated systems as obtained from adsorption isotherms

\begin{tabular}{|c|c|c|c|c|c|}
\hline $\begin{array}{l}\text { Molecules on gold } \\
\text { surface }\end{array}$ & $\begin{array}{l}\text { Monolayer capacity, } \\
\Gamma_{\mathrm{m}} / \mathrm{nmol} \mathrm{cm}^{-2} \text { Eq. }(5)\end{array}$ & $\begin{array}{l}\text { Cross sectional area, } \\
a_{\mathrm{m} .} / \mathrm{nm}^{2} \text { Eq. (6) }\end{array}$ & $a_{\mathrm{m}} / a_{\mathrm{m}, \mathrm{calc}}$ & $\begin{array}{l}\text { Calculated cross sectional } \\
\text { area } * a_{\mathrm{m}, \text { calc }} / \mathrm{nm}^{2}\end{array}$ & $\begin{array}{c}\text { Surface } \\
\text { orientation }\end{array}$ \\
\hline L-Cysteine & 0.325 & 0.513 & 1.425 & 0.360 & parallel \\
\hline L-Glutathione & 0.135 & 1.234 & 1.505 & 0.820 & parallel \\
\hline Ibuprofen & 0.330 & 0.505 & 0.789 & 0.640 & parallel \\
\hline Dopamine & 0.860 & 0.194 & 0.359 & 0.540 & perpendicular \\
\hline
\end{tabular}

* The $a_{\mathrm{m} \text {,calc }}$ is the cross sectional area calculated by the MarvinSketch ${ }^{27}$ program based on the conformation of molecules.

Table 2. The maximal adsorption capacities $\left(\Gamma_{\mathrm{m}}\right)$ and molecular cross sectional areas $\left(a_{\mathrm{m}}\right)$ on functionalized gold surface for different bioconjugated systems

\begin{tabular}{lccccc}
\hline $\begin{array}{l}\text { Molecules on functiona- } \\
\text { lized gold surface }\end{array}$ & $\begin{array}{c}\text { Adsorptioncapacity, } \\
\Gamma_{\mathrm{m} .} / \mathrm{nmol} \mathrm{cm}^{-2} \text { Eq. (5) }\end{array}$ & $\begin{array}{c}\text { Cross sectional area, } \\
a_{\mathrm{m}} / \mathrm{nm}^{2} \mathrm{Eq} .(6)\end{array}$ & $\begin{array}{c}a_{\mathrm{m}} / a_{\mathrm{m} \text {,calc }} \\
\text { L-Cys-Ibuprofen }\end{array}$ & $\begin{array}{c}\text { Calculated cross section } \\
\text { area, } * a_{\mathrm{m}, \text { calc }} / \mathrm{nm}^{2}\end{array}$ & $\begin{array}{c}\text { Surface } \\
\text { orientation }\end{array}$ \\
L-GSH-Ibuprofen & 0.180 & 0.513 & 0.801 & 0.640 & parallel \\
p-Cys-Dopamine & 0.640 & 0.926 & 1.447 & 0.640 & parallel \\
L-GSH-Dopamine & 0.580 & 0.260 & 0.481 & 0.540 & perpendicular \\
\hline
\end{tabular}

* The $a_{\mathrm{m} \text {,calc }}$ is the cross sectional area calculated by the MarvinSketch ${ }^{27}$ program based on the conformation of molecules. 


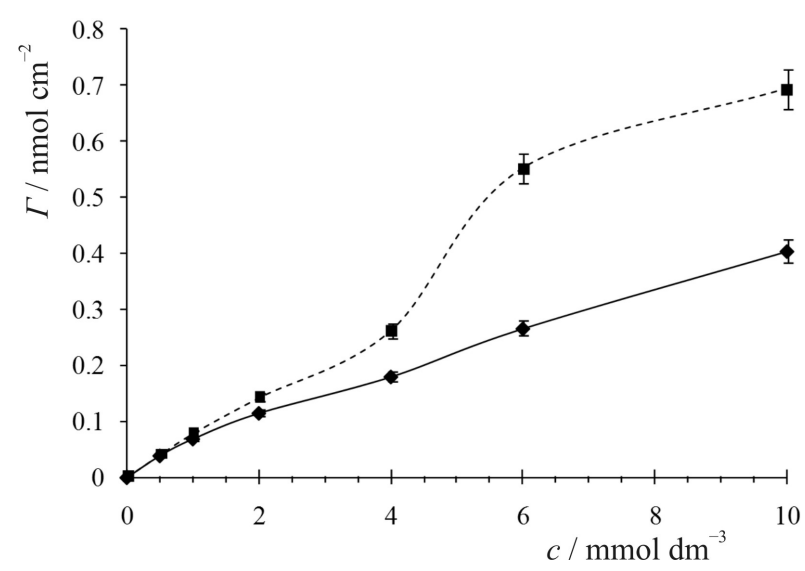

Figure 6. Adsorption isotherms for ibuprofen ( $\downarrow)$ and dopamine (a) on gold surface from aqueous solutions. The average error of $\Gamma$ values is $\pm 6.8 \%$, depending on the concentration range.

$$
\frac{1}{\Gamma}=\frac{1}{\Gamma_{\mathrm{m}}}+\frac{1}{K \cdot c}
$$

where $c$ is concentration expressed as amount (number of moles) per volume of the solution, $\Gamma$ denotes surface concentration expressed as amount (number of moles) per surface area at equilibrium concentration of adsorbent $c$, while $\Gamma_{\mathrm{m}}$ represents complete surface coverage (i.e. monolayer adsorption capacity) and $K$ is the equilibrium constant of adsorption.

In another form Eq. (6) reads

$$
\frac{c}{\Gamma}=\frac{1}{K}+\frac{1}{\Gamma_{\mathrm{m}}} \cdot c
$$

Accordingly, the slope of the plot $c / \Gamma v s . c$ yields reciprocal value of the monolayer surface concentration $\Gamma_{\mathrm{m}}$ being related to the cross-sectional area of adsorbed molecules $a_{\mathrm{m}}$ by Eq. (4).

The linearized adsorption isotherms for ibuprofen and dopamine at lower concentrations are presented in Figure 7 providing the monolayer adsorption capacities and corresponding cross-sectional areas, as well as adsorption equilibrium constants. For ibuprofen: $\Gamma_{\mathrm{m}}=0.33$ nmol cm ${ }^{-2} ; a_{\mathrm{m}}=0.505 \mathrm{~nm}^{2}, K=9.2 \times 10^{-5} \mathrm{~cm}$. For dopamine: $\Gamma_{\mathrm{m}}=0.86 \mathrm{nmol} \mathrm{cm}{ }^{-2} ; a_{\mathrm{m}}=0.194 \mathrm{~nm}^{2}, K=$ $8.6 \times 10^{-5} \mathrm{~cm}$. The results are summarized in Table 1 .

\section{Adsorption on Functionalized Gold Thin Films}

Since ibuprofen is bound to the surface of gold just by reversible physical adsorption, let us examine how the binding of ibuprofen on gold can be improved by functionalization of gold thin film by L-cysteine. First part of the experiment presented in Figure 8 corresponds to functionalization of the gold surface by L-cysteine, the concentration of which was gradually increased up to 10

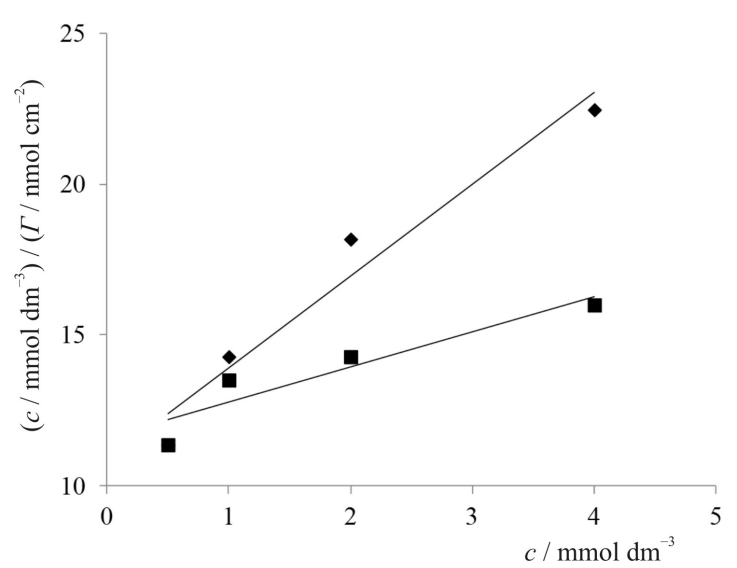

Figure 7. The linear representation of the adsorption isotherms at lower surface concentration of ibuprofen $(\downarrow$, slope

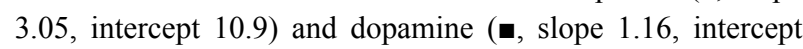
11.6) on gold surface.

mmol $\mathrm{dm}^{-3}$. After washing with water the irreversibly bound L-cysteine molecules remained. The surface concentration of irreversibly bound L-cysteine was found to be $0.5 \mathrm{nmol} \mathrm{cm}$. In the second part of the experiment ibuprofen was introduced in raising concen-

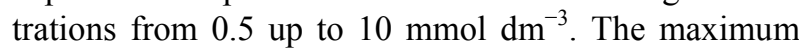
surface concentration of ibuprofen was obtained to be 1 nmol cm${ }^{-2}$. After the washing step the ibuprofen surface concentration was reduced to $0.5 \mathrm{nmol} \mathrm{cm}$, which is just equal to the surface concentration of irreversibly bound L-cysteine.

In the experiments the adsorption of ibuprofen and dopamine on the functionalized gold surface was examined. The gold surface was functionalized with Lcysteine or L-glutathione in concentration of $1 \mathrm{mmol}$ $\mathrm{dm}^{-3}$. This concentration was selected for surface functionalization, because at the higher concentrations (10 mmol $\mathrm{dm}^{-3}$ ) adsorbent is bound only by physical adsorption, which can be seen in Figure 3. After washing step, the irreversibly bound L-cysteine or L-glutathione remained bound. Surface concentration of L-cysteine was $0.325 \mathrm{nmol} \mathrm{cm}$ and of L-glutathione was 0.135 nmol cm${ }^{-2}$. The maximum surface concentration of ibuprofen adsorbed on gold functionalized by L-cysteine

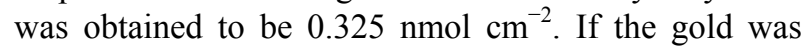
functionalized by L-glutathione the lower coverage of ibuprofen was observed, i.e. $0.180 \mathrm{nmol} \mathrm{cm}^{-2}$ adsorbed (see Figure 9).

Adsorption isotherms for adsorption of ibuprofen and dopamine on L-cysteine and L-glutathione modified gold are presented in Figures 9 and 10, respectively.

In addition the values based on the conformation of molecules, calculated using the MarvinSketch program, ${ }^{27}$ are presented in Tables 1 and 2 . These data provide information on the molecular arrangements on the flat surface by comparing calculated and the experimental data. (See the $a_{\mathrm{m}} / a_{\mathrm{m} \text {,calc }}$ ratio in Tables 1 and 2). 


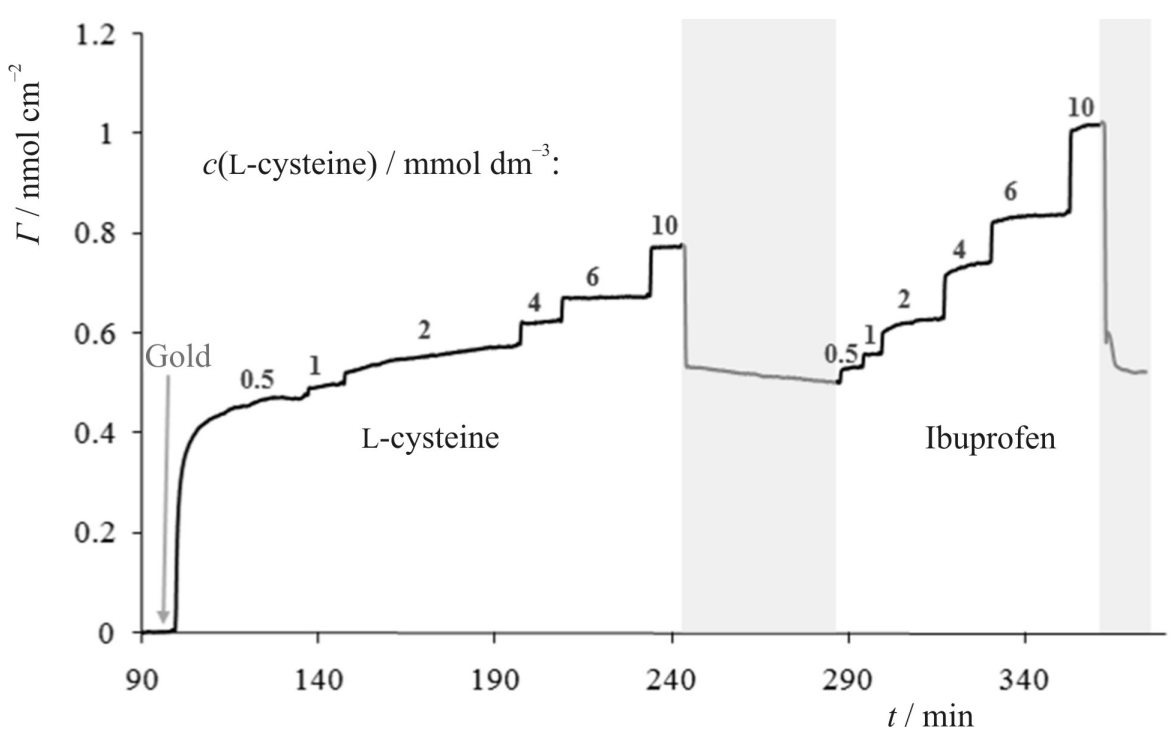

Figure 8. Representative SPR curves (sensorgrams) of adsorption of ibuprofen on L-cysteine functionalized gold surface from aqueous solutions. (at concentrations of $0.5,1,2,4,6,10 \mathrm{mmol} \mathrm{dm}^{-3}$ ). Washing with water is denoted by gray areas.

The minimal and the maximal cross-sectional areas of each molecule in each system were calculated using the MarvinSketch ${ }^{27}$ software. Comparing the maximal and the minimal cross-sectional data with the values obtained from measurements it is possible to gain information about the surface orientation of a molecule. Accordingly, it may be concluded that adsorbed cysteine and the glutathione molecules are parallel with respect to the gold surface. The cysteine could be covalently bound to the gold surface (by $\mathrm{Au}-\mathrm{S}$ bond). ${ }^{28}$ The $\mathrm{pH}$ values of aqueous ibuprofen and the dopamine solutions can be found between 6 and 6.5 , at which the cysteine is in a zwitterionic form. Due to this zwitterionic configuration cysteine can interact with the deprotonat-

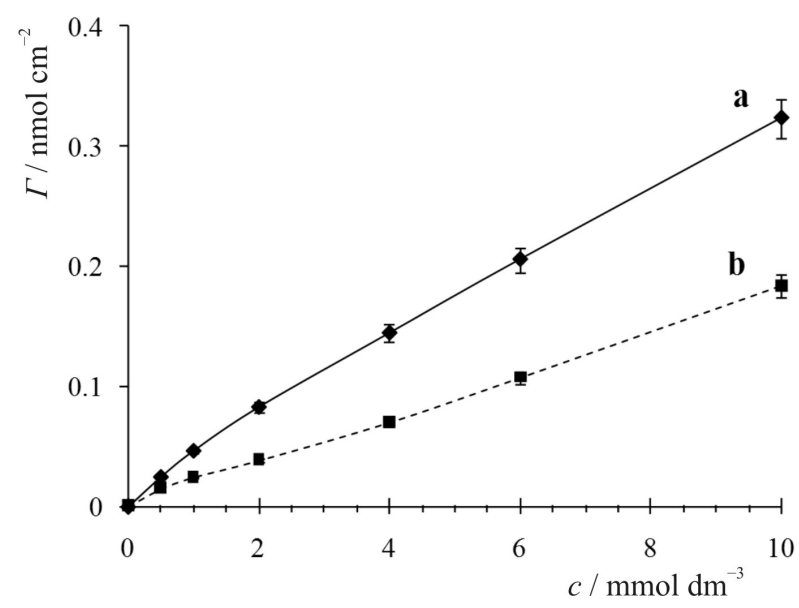

Figure 9. Adsorption isotherms of ibuprofen on gold surface from aqueous solutions functionalized by $1 \mathrm{mmol} \mathrm{dm}^{-3} \mathrm{~L}$ cysteine (a) and L-glutathione (b). The average error of $I$ values is $\pm 5.2 \%$ depending on the solution cocncentration. ed form of the ibuprofen through the amino group of the cysteine, and the protonated form of the dopamine with the carboxyl group of the cysteine.

In order to obtain information on the adsorption binding energies, the temperature dependency of the adsorption isotherms were determined, as shown in Figures 11 and 12.

The analysis of data presented in Figures 11 and 12 was performed on the basis of Eqs. $(8,9){ }^{29}$

$$
R T \ln K=-\Delta H+T \Delta S
$$

where $R$ is the gas constant, while $\Delta H$ and $\Delta S$ denote reaction enthalpy and entropy, respectively.

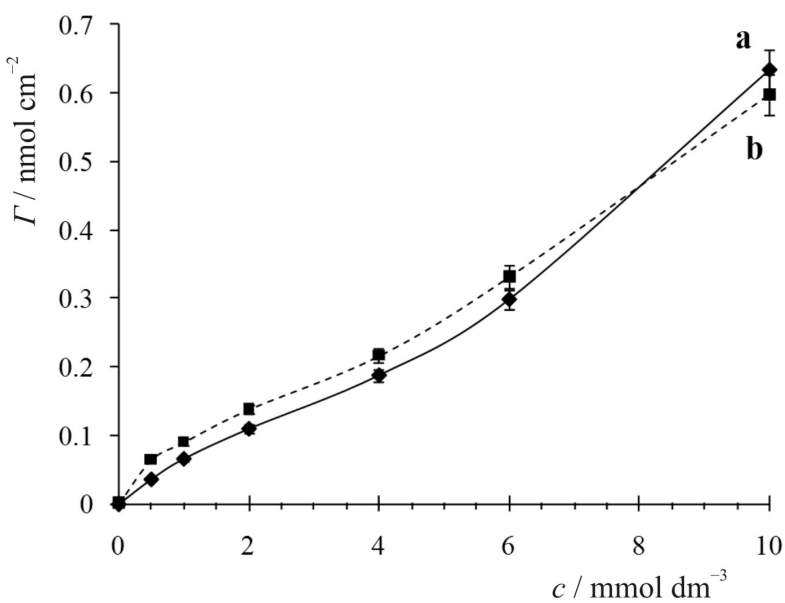

Figure 10. Adsorption isotherms of dopamine on gold surface from aqueous solutions functionalized by $1 \mathrm{mmol} \mathrm{dm}^{-3} \mathrm{~L}$ cysteine (a) and L-glutathione (b). The average error of $I$ values is $\pm 5.2 \%$ depending on the solution concentration. 


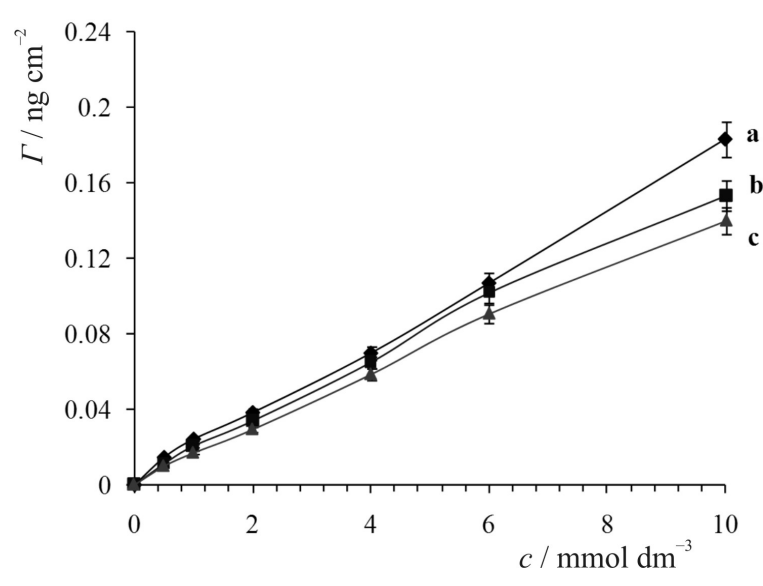

Figure 11. Temperature dependency of the L-glutathione/ibuprofen adsorption isotherms at (a) $20^{\circ} \mathrm{C}$, (b) $25^{\circ} \mathrm{C}$ and (c) $30^{\circ} \mathrm{C}$ from aqueous solutions. The error of $\Gamma$ values is \pm 5.2 $\%$. depending on the solution concentration.

According to Eqs. $(6,8)$ the adsorption enthalpy (isosteric heat of adsorption) could be determined from the temperature dependency of the equilibrium concentration of the adsorbent in the bulk of the solution at constant surface concentration (surface coverage) as

$$
\Delta H=-R\left(\frac{d(\ln c)}{d(1 / T)}\right)_{\Gamma}
$$

Results are presented in the Table 3.

The values of adsorption heats (enthalpies) were calculated by Eq. (9), at chosen surface concentrations, assuming linearity of $\ln c\left(T^{-1}\right)$ function. The surface coverage $\left(\Theta=\Gamma / \Gamma_{\max }\right)$ for ibuprofen is lower with respect to monolayer coverage so that molecular interactions are less pronounced, with respect to the case of dopamine coupling on L-glutathione functionalized surface. Less negative adsorption enthalpies could be measured if the adsorbed molecules repel each other. If the molecules displace each other on the gold surface, they will adsorb in a disordered layer, causing less negative adsorption enthalpies at higher surface coverage. ${ }^{30}$ For adsorption of dopamine on L-glutathione modified gold surface

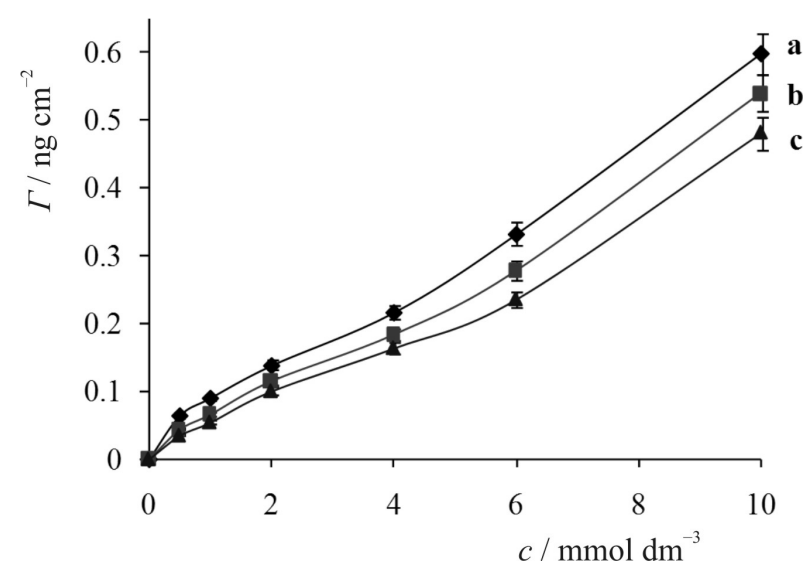

Figure 12. Temperature dependency of the L-glutathione/dopamine adsorption isotherms at (a) $20^{\circ} \mathrm{C}$, (b) $25^{\circ} \mathrm{C}$ and (c) $30{ }^{\circ} \mathrm{C}$ from aqueous solutions. The error of $\Gamma$ values is \pm 5.2 $\%$ depending on the solution concentration.

higher exothermic intermolecular interactions were found at lower surface coverage, which is in accordance with the fact that dopamine molecules show very strong binding to the functionalized surface having an perpendicular molecular orientation. (See Figure 13.)

\section{DISCUSSION}

Adsorption of ibuprofen on gold surface in the flow system exhibits almost full reversibility, suggesting that the adsorption is of the physical type. However, adsorption of dopamine on the surface of gold is only partially reversible. Figure 7 represents corresponding adsorption isotherms', suggesting that adsorption of dopamine is described by a two-stage isotherm. It may be concluded that first dopamine layer is irreversibly bound while the second layer is anchored by physical forces. In Figure 8 the linear representation of the adsorption isotherms based on Eq. (5) is presented yielding the crosssectional areas of the adsorbed ibuprofen and dopamine molecules. Since adsorbed amounts are significantly higher than the monomolecular surface concentration one can conclude that more than one layer of molecules are adsorbed on the gold surface.

Table 3. Adsorption enthalpies $(\Delta H)$ for L-glutathione functionalized gold surface coupled with ibuprofen and dopamine molecules as a function of surface concentration $(\Gamma)$ and surface coverage $(\Theta)$

\begin{tabular}{cccccc}
\hline \multicolumn{2}{c}{ L-glutathione-ibuprofen coupling } & \multicolumn{3}{c}{ L-glutathione-dopamine coupling } \\
$\Gamma / \mathrm{nmol} \mathrm{cm}^{-2}$ & $\Theta$ & $\Delta H / \mathrm{kJ} \mathrm{mol}^{-1}$ & $\Gamma / \mathrm{nmol} \mathrm{cm}^{-2}$ & $\Theta$ & $\Delta H / \mathrm{kJ} \mathrm{mol}^{-1}$ \\
\hline 0.005 & 0.028 & -29.9 & 0.007 & 0.012 & -51.2 \\
0.024 & 0.133 & -24.9 & 0.033 & 0.057 & -46.4 \\
0.044 & 0.244 & -18.3 & 0.059 & 0.101 & -43.0 \\
0.068 & 0.377 & -11.9 & 0.091 & 0.156 & -40.4 \\
\hline
\end{tabular}


(a) Dopamine

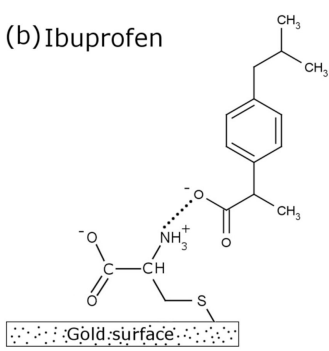

Figure 13. The schematic illustration of the interactions between the cysteine-dopamine (a) and the cysteine-ibuprofen (b) systems (see Ref. 28).

It is shown that L-cysteine adsorption increases stepwise, and that after desorption stage adsorption is reduced to about $40-50 \%$ of the original value. This finding suggests chemical interaction developed between thiol groups of cysteine molecules and the gold surface. To such a surface, ibuprofen molecules were bound at four different concentrations.

According to results presented in this study, it can be concluded that in case of functionalization by cysteine, dopamine is bound to the surface in larger amounts than ibuprofen. Such a finding could be expected since dopamine contains amino groups enabling formation of a stronger chemical bond with the cysteine molecule, with respect to ibuprofen. Adsorbed cysteine molecules are oriented parallel to the surface, while bounded dopamine molecules are oriented perpendicular to the surface in contrast to ibuprofen exhibiting parallel orientation. Glutathione as a functionalization agent is characterized by a parallel orientation to the gold surface, while bounded dopamine are oriented perpendicular, again in contrast to ibuprofen being bounded parallel with respect to the surface (Table 2). In the case of Au-L-glutathione-dopamine coupling system the adsorbed amount, corresponding to the monomolecular coverage, does not differ significantly from $\mathrm{Au}$ dopamine system. But these adsorbed amounts are still higher than for Au-L-cysteine-ibuprofen or Au-Lglutathione-ibuprofen systems. There are stronger molecular interactions, probably due to interaction of amino group in the dopamine with two carboxylic groups of L-glutathione molecule which is anchored to the gold surface by the SH-group; $\Delta H=-40 . .-50 \mathrm{~kJ} \mathrm{~mol}^{-1}$ (Table 3). The interaction with ibuprofen molecules are les pronounced $\left(\Delta H=-11 \ldots-30 \mathrm{~kJ} \mathrm{~mol}^{-1}\right)$, because the carboxylic group can interact only with the amino group of L-glutathione. (See the molecular structures of dopamine and ibuprofen presented in Figure 13.)

The temperature dependence of ibuprofen adsorption on gold surfaces functionalized by glutathione was not found to be significantly pronounced which suggest lower initial energies of interaction as compared to the glutathione/dopamine system.
Table 3 reveals that the glutathione/dopamine interaction is significantly stronger than the glutathione/ibuprofen interaction. As surface coverage increases, values of the isosteric heat of adsorption indicate increasingly stronger interactions: increase of surface coverage enables a stronger linkage between glutathione and dopamine molecules than between glutathione and ibuprofen. Less negative enthalpies could be measured if the adsorbed molecules displace each other at the gold surface. In such a case the molecular orientation would be partially disordered causing less negative adsorption enthalpy at higher surface coverage. ${ }^{30}$ Using the data from Table 2 and 3, the coupling effect between ibuprofen-L-glutathione system at maximum adsorbed amount, expressed as the molecular coupling ratio, is $37 \%$ for ibuprofen, a⿶d45 \% for dopamine -Lglutathione system.

As the results of our study summarized in discussion represent that the surface plasmon resonance spectroscopy is one of the best suitable two-dimensional technique to determine the adsorption capability of biomolecules on "pure" or biofunctionalized gold surface from aqueous solutions.. Among this technique the other possible method for characterization of adsorption is the Optical Waveguide Lightmode Spectroscopy (OWLS).

Acknowledgements. This research was supported by the European Union and the State of Hungary, co-financed by the European Social Fund in the framework of TÁMOP 4.2.4. A/2-11-1-2012-0001 'National Excellence Program', TÁMOP-4.2.2.A-11/1/KONV-2012-0047. The authors are also thankful for the financial support of the bilateral agreements between the Hungarian Academy of Sciences and the Croatian Academy of Sciences and Art.

\section{REFERENCES}

1. C. Nylander, B. Liedberg, and T. Lind, Sensor. Actuator. 3 (1982-1983) 79-88.

2. B. Liedberg, C. Nylander, and I. Lunström, Sensor. Actuator. 4 (1983) 299-304.

3. A. Nooke, U. Beck, A. Hertwig, A. Krause, H. Krüger, V. Lohse, D. Negendank, and J. Steinbach, Sensor. Actuat. B-Chem. 149 (2010) 194-198.

4. M.G. Manera, G. Leo, M.L. Curri, P.D. Cozzoli, R. Rella, P. Siciliano, A. Agostiano, and L. Vasanelli, Sensor. Actuat. B-Chem. 100 (2004) 75-80.

5. M. Piliarik, H. Vaisocherová, and J. Homola, Biosens. Bioelectron. 20 (2005) 2104-2110.

6. K. Pimkova, M. Bockova, R. Kotlin, J. Suttnar, J. Homola, and J. E. Dyr, Leukemia Res. 35 (2011) S50.

7. H. Wang, J. Shi, Y. Wang, K. Cai, Q. Wang, X. Hou, W. Guo, and F. Zhang, J. Pharmaceut. Biomed. 50 (2009) 1026-1029.

8. R.W. Wood, Proc. Phys. Soc. London 18 (1902) 269-275

9. U. Fano, J. Opt. Soc. Am. 31 (1941) 213-222.

10. A. Z. Otto, Phys. 216 (1968) 398-410.

11. E. Kretschmann and H. Z. Raether, Naturforsch. 23 (1968) 2135-2136 
12. W. W. Lam, L. H. Chu, C. L. Wong, and Y. T. Zhang, Sensor. Actuat. B-Chem. 105 (2005) 138-143.

13. B. Špačková, M. Piliarik, P. Kvasnička, C. Themistos, M. Rajarajan, and J. Homola, Sensor. Actuat. B-Chem. 139 (2009) 199-203.

14. E. Pál, V. Hornok, D. Sebők, A. Majzik, and I. Dékány, Colloid Surface B 79 (2010) 276-283.

15. P. Adam, J. Dostálek, and J. Homola, Sensor. Actuat. B-Chem. 113 (2006) 774-781.

16. J. Dostálek, J. Homola, and M. Miler, Sensor. Actuat. B-Chem. 107 (2005) 154-161.

17. J. Homola, Chem. Rev. 108 (2008) 462-493.

18. J. F. Hainfeld, D. N. Slatkin, T. M. Focella, and H. M. Smilowitz, Brit. J. Radiol. 79 (2006) 248-253.

19. M. M. van Schooneveld, D. P. Cormode, R. Koole, J. T. van Wijngaarden, C. Calcagno, T. Skajaa, J. Hilhorst, D. C. 't Hart, Z. A. Fayad, W. J. M. Mulder, and A. Meijerink, Contrast Media Mol. I 5 (2010) 231-236.

20. J. F. Hainfeld, D. N. Slatkin, T. M. Focella, and H. M. Smilowitz, Brit. J. Radiol. 79 (2006) 248-253.
21. X. Huang, M. A. El-Sayed, J. Adv. Res. 1 (2010) 13-28.

22. A. M. Alkilany, L. B. Thompson, S. P. Boulos, P. N. Sisco, and C. J. Murphy, Adv. Drug Deliver. Rev. 64 (2012) 190-199.

23. S. Rana, A. Bajaj, R. Mout, and V. M. Rotello, Adv. Drug Deliver. Rev. 64 (2012) 200-216.

24. A. Majzik, L. Fülöp, E. Csapó, F. Bogár, T. Martinek, B. Penke, G. Bíró, and I. Dékány, Coll. Surf. B 81 (2010) 235-241.

25. Á. Veres, T. Rica, L. Janovák, M. Dömök, N. Buzás, V. Zöllmer, T. Seemann, A. Richardt, and I. Dékány, Catal. Today 181 (2012) 156-162.

26. E. Csapó, R. Patakfalvi, V. Hornok, L. T. Tóth, Á. Sipos, A. Szalai, M. Csete, and I. Dékány, Coll. Surf. B 98 (2012) 43-49.

27. MarviSketch, http:/www.chemaxon.com/products/marvin/marvinsketch/

28. H. Häkkinen, Nature Chemistry 4 (2012) 443-455

29. V.C. Srivastava, I. D. Mall, and I. M. Mishra, Chem. Eng. J. 132 (2007) 267-278

30. J. Dion, S. Seminar, Adsorption of Molecules onto Metallic Surfaces: Theory and Applications,

http://www.uvm.edu/ jdion/personal/adsorption.html 